\title{
Measurement and analysis of the impact of transverse incoherent wakefields in a light source storage ring
}

\author{
P. Brunelle, R. Nagaoka, and R. Sreedharan \\ Synchrotron SOLEIL, L'Orme des Merisiers, Saint-Aubin, Gif-sur-Yvette 91192, France
}

(Received 28 July 2015; published 15 April 2016)

\begin{abstract}
The influence of incoherent wakefields arising from a high current beam circulating in a ring composed of many flat chambers on the beam is analyzed numerically and experimentally for the SOLEIL storage ring. Three distinct ranges of the excited field are studied: the long multiturn regime in multibunch fillings, the medium regime involving neighboring bunches, and the short intrabunch regime. Two theoretical models, which both take into account the field diffusion across chamber walls of finite thickness, are employed to compare the measurement with theoretical expectations. Specific experimental methods, based respectively upon orbit analysis, bunch-by-bunch transverse feedback and a single particle betatron resonance, are introduced to measure the incoherent focusing effect in the three regimes. It is found that the excited incoherent fields can be large enough to affect the ring performance. The non-negligible contribution of nonevaporable getter coating is shown to improve the agreement between measurements and calculations.
\end{abstract}

DOI: 10.1103/PhysRevAccelBeams.19.044401

\section{INTRODUCTION}

The SOLEIL storage ring is the French third generation synchrotron light source operating at the energy of $2.75 \mathrm{GeV}$. High brilliance photon beams are generated mainly with insertion devices (ID) in the VUV to hard $x-$ ray energy range. Up to five different modes of operation are delivered to users in top-up operation. The maximum stored beam current is $500 \mathrm{~mA}$ in the multibunch $4 / 4$ filling pattern (416 bunches). The daily operation mode is the multibunch hybrid filling pattern ( $445 \mathrm{~mA}$ in 312 bunches and $5 \mathrm{~mA}$ in a single bunch). Two other high bunch current modes $(1 \times 15 \mathrm{~mA}$ and $8 \times 12.5 \mathrm{~mA})$ are also provided. The compact $354 \mathrm{~m}$ long magnetic structure of the ring integrates 24 straight sections (SSs), mostly dedicated to IDs with small gap chambers. The full vertical aperture of the standard aluminum vacuum chamber (for quadrupoles and sextupoles) is $25 \mathrm{~mm}$ and it reduces to $14 \mathrm{~mm}$ in the long SSs and $10 \mathrm{~mm}$ in the medium SSs. In addition, six $1.8 \mathrm{~m}$ long in-vacuum undulators are installed in short SSs with a $5.5 \mathrm{~mm}$ minimum magnetic gap. Figure 1 shows a typical SS vacuum chamber cross section. The internal apertures of each type of vacuum chamber are summarized in Table I. The storage ring optics went through a major upgrade in the year $2012[3,4]$ and the main storage ring parameters can be found at the SOLEIL web site ${ }^{1}$.

*pascale.brunelle@synchrotron-soleil.fr

${ }^{1}$ www.synchrotron-soleil.fr.

Published by the American Physical Society under the terms of the Creative Commons Attribution 3.0 License. Further distribution of this work must maintain attribution to the author(s) and the published article's title, journal citation, and DOI.
A high intensity beam experiences the so-called incoherent current-dependent transverse focusing due to the proximity of metallic walls of vertically reduced chambers. The beam is then potentially under strong resistive-wall fields. The usual transverse dipolar wake that can drive a beam unstable is excited when the beam center of mass is displaced with respect to the design orbit. The noncompensated resistive-wall field remains in a flat chamber, even with a nondisplaced beam, due to the noncircular geometry of the cross section. The main component of the residual field above is known to be quadrupolar for a trailing particle, acting on it incoherently similar to a quadrupole magnet. The difference is that its focusing strength depends linearly on the beam current, giving rise to a currentdependent betatron tune shift in the two transverse planes. For this reason, the wakefield is often called a quadrupolar wake, while it is also called a monopole wake upon the fact that a monopole beam excites it. Here we shall refer to it as an incoherent wake due to the absence of coherence in the excited field as well as to the incoherent response of a

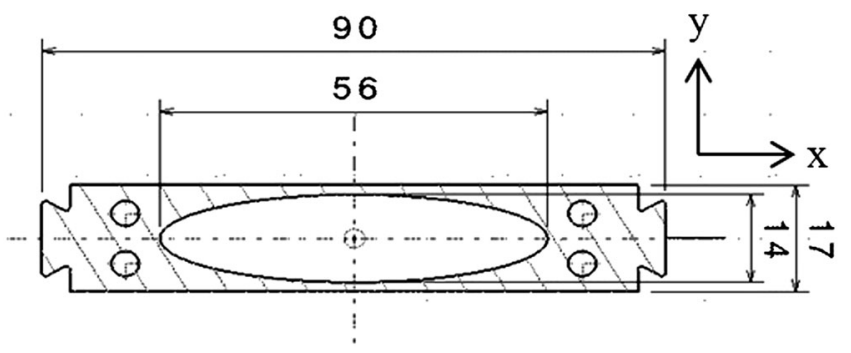

FIG. 1. Typical cross section of vacuum chambers in straight sections. Dimensions are given in $\mathrm{mm}$ and correspond to the case in the long SSs. 
TABLE I. The exact values $\partial W_{x} / \partial x_{w}$ (evaluated at $z=1 \mathrm{~m}$ ) obtained with the scheme [1] and those via the analytical formula for parallel plates [2] for SOLEIL chambers. $a$ : horizontal half aperture; $b$ : vertical half aperture; $d$ : chamber thickness at $x=0$; Al: aluminum $\left(\rho_{R}=2.8 \times 10^{-8} \Omega \mathrm{m}\right)$; StSt: stainless steel $\left(\rho_{R}=100 \times 10^{-8} \Omega \mathrm{m}\right)$.

\begin{tabular}{|c|c|c|c|c|c|c|c|}
\hline \multirow[b]{2}{*}{ Chamber type } & \multirow[b]{2}{*}{$a(\mathrm{~mm})$} & \multirow[b]{2}{*}{$b(\mathrm{~mm})$} & \multirow[b]{2}{*}{$d(\mathrm{~mm})$} & \multirow[b]{2}{*}{ Material } & \multicolumn{2}{|c|}{$\left(\partial W_{x} / \partial x_{w}\right)\left(10^{12} \mathrm{VC}^{-1} \mathrm{~m}^{-2}\right)$} & \multirow[b]{2}{*}{$(a-b) /(a+b)$} \\
\hline & & & & & Parallel plates & Yokoya scheme & \\
\hline Standard Al chamber & 35 & 12.5 & 2 & $\mathrm{Al}$ & 0.0368 & 0.0363 & 0.47 \\
\hline Dipole StSt chamber & 35 & 12.5 & 2 & StSt & 0.2200 & 0.2170 & 0.47 \\
\hline rf cavity ( 2 nd outer) taper & 42.5 & 31.25 & 1 & StSt & 0.0141 & 0.0100 & 0.15 \\
\hline Medium SS taper & 29 & 8.75 & 2 & StSt & 0.6414 & 0.6438 & 0.54 \\
\hline Medium SS chamber & 23 & 5 & 2 & $\mathrm{Al}$ & 0.5752 & 0.5990 & 0.64 \\
\hline Long SS taper & 32.5 & 4.75 & 2 & StSt & 0.4636 & 0.4647 & 0.54 \\
\hline Long SS chamber & 28 & 7 & 2 & $\mathrm{Al}$ & 0.2096 & 0.2147 & 0.60 \\
\hline
\end{tabular}

beam. The characteristics of the incoherent wakefields were already studied for different chamber cross section geometries $[1,5,6]$. The concerned tune shifts were observed in a number of storage rings such as PEP-II, ESRF, BESSY, KEKB and SOLEIL [2,7-10] and theoretical studies were made to reproduce the measurement, addressing the importance of diffusion of the excited fields across metallic chamber walls of finite thickness $[2,7,10]$.

At SOLEIL, the magnitude of incoherent tune shifts arising from the chamber cross section asymmetry was estimated in multibunch and single bunch at the design phase [11]. The scope of this paper is to present the extensive experimental studies made to characterize the incoherent tune shifts and beta beatings arising from the incoherent wakefields. The measurement and the analysis of the data were carried out using specific tools and methods such as orbit response matrix analysis for optics tuning, a bunch-bybunch transverse feedback (TFB) system, and exposure of the beam to a betatron resonance. While the first method allowed identification of the location and the strength of wakefields, the latter two helped explore the short-range nature of the excited wakefields by measuring the incoherent tunes of a single high intensity bunch and of weak intensity bunches as a function of the distance from an intense bunch. A comparison with existing models will be presented.

The paper is organized as follows: in Sec. II, we shall first review the theory developed for incoherent wakefields and describe the ways the effects are numerically evaluated in this paper. In Sec. III, the long-range nature of the wakefield is addressed by performing the measurement and analysis in multibunch fillings, where in particular the impact of in-vacuum ID gap closure shall be described. Then in Sec. IV, the tune shifts of weak parasitic bunches are measured as a function of their distance from a high intensity bunch using a bunch-by-bunch diagnostics and are compared with expectations. In Sec. V, the incoherent tunes of a high intensity bunch are measured indirectly by making use of a betatron coupling resonance. The influence of bunch lengthening as well as that of the nonevaporable getter (NEG) coating is discussed. Finally, a conclusion is given in Sec. VI.

\section{REVIEW OF THE THEORY AND NUMERICAL EVALUATION OF INCOHERENT WAKEFIELD EFFECTS}

Theoretical studies made in the past may be viewed as consisting of two parts. First, the amplitude of the excited incoherent wakes was investigated as a function of cross section geometries for resistive-wall chambers of infinite thickness, where it was analytically derived by Gluckstern, van Zeijts and Zotter for elliptical and rectangular cross sections [5]. Yokoya developed a formalism to compute numerically the focusing strength for an arbitrary cross section [1]. More recently, Mounet and Métral made an extension to treat nonrelativistic beams in a flat chamber made of layers of any materials [12]. Here the incoherent wake $\frac{\partial W_{x}}{\partial x_{w}}$ can be introduced as an additional acceleration in the horizontal equation of motion of a witness particle following the wake-inducing source particle at a distance $z$ behind as

$$
x_{w}^{\prime \prime}=\frac{e}{E / e} \frac{\partial W_{x}}{\partial x_{w}} \cdot x_{w}=-k_{\mathrm{eff}}^{e}(z) \cdot x_{w},
$$

where $x_{w}$ denotes the horizontal betatron displacement of the witness particle, the double prime a double derivative with respect to the longitudinal coordinate $s, e$ the electron charge, $E$ the particle energy and $k_{\mathrm{eff}}^{e}(z)$ is the effective focusing strength of the vacuum chamber per unit length induced by the passage of a single electron. Note that we define $\frac{\partial W_{x}}{\partial x_{w}}$ per unit length with its dimension $\mathrm{VC}^{-1} \mathrm{~m}^{-2}$, to be able to evaluate it piecewise to obtain its integrated effect around the machine. The effective focusing strength $k_{\mathrm{eff}}^{e}(z)$ for a flat chamber is known to be approximately given by the following expression derived for a pair of horizontally parallel plates [2]:

$$
k_{\mathrm{eff}}^{e}(z)=\frac{\pi^{2}}{24} \frac{e}{E / e} \frac{c \sqrt{Z_{0} \rho_{R}}}{\pi^{3 / 2} b^{3}} \frac{1}{z^{1 / 2}},
$$

where $c$ is the speed of light, $Z_{0}(=376.7 \Omega)$ the vacuum impedance, $\rho_{R}$ the resistivity of the chamber wall, and $b$ is the half aperture of the parallel plates. The quantity $\pi^{2} / 24$ 
appearing on the rhs of Eq. (2) represents the geometric factor for parallel plates [13]. The vertical equation of motion is then identical to the horizontal one but has a minus sign on the rhs. Then a particle is focused horizontally and defocused vertically in a horizontally elongated chamber. In the present study, we employed Yokoya's formalism to compute the incoherent wake function $\frac{\partial W_{x}}{\partial x_{w}}$ of a chamber of arbitrary cross section. A computer code written in $\mathrm{C}$ was prepared accordingly [8], which can be exported upon request. Numerical values obtained for the different types of SOLEIL vacuum chambers are however found to be sufficiently close to those given by the analytical parallel plates formula in Eq. (2) (Table I).

The second part addresses the time dependence of the excited wakes. With the $z^{-1 / 2}$ dependence that originates from an infinitely thick resistive wall in Eq. (2), the accumulated monopole field diverges with successive beam passages. The standard infinitely thick wall model cannot therefore explain the finite incoherent betatron tune shifts. The physical importance of field penetration across a finitely thick wall in describing the tune shifts was first pointed out by Heifets [7]. Chao, Heifets and Zotter developed a model (referred in this paper to as model A) that incorporates the effect of field penetration by assuming that the time dependence of field diffusion in a general resistive-wall chamber with finite wall thickness is sufficiently close to that analytically known for a cylindrical chamber [2]. Employing the smooth approximation for the linear optics, their analytically derived formula for the slopes of the tune shifts with current in the two transverse planes reads

$\frac{d \nu_{x, y}}{d I}= \pm\left(\frac{\pi r}{48 \nu_{x, y}}\right)\left(\frac{Z_{0}}{E / e}\right)\left(\frac{R}{b}\right)^{2}\left(\frac{L}{C}\right), \quad r=1+\frac{b^{2}}{D^{2}}$

Here, $\nu_{x}\left(\nu_{y}\right)$ denotes the horizontal (vertical) betatron tune, $I$ the beam current, $C$ the ring circumference, $R$ the ring radius, and $L$ represents the portion of the ring where flat chambers are installed. The parameter $r$ is dependent on $D$, the distance from the beam axis to the outer magnet wall, and $r$ is unity in case there is no outer wall $(D=\infty)$. As well discussed in their paper, the derived tune shifts formula in Eq. (3) does not depend on the chamber wall thickness or the resistivity. This result is justified upon the fact that the concerned tune shifts are nothing else but the Laslett tune shifts, and their work can thus be considered as an extension of the Laslett's work to resistive-wall chambers.

Another model was developed by Shobuda and Yokoya (referred to as model B in this paper) for tune shifts on the basis of the previous work of Yokoya. They solved directly the Maxwell equations with a finite thick metallic wall in one dimension (i.e. a flat chamber) by assuming that the radius of the beam pipe $b$ is sufficiently large compared to
TABLE II. Incoherent tune shifts calculated for SOLEIL in the $4 / 4$ filling at a beam current of $500 \mathrm{~mA}$. Numbers without brackets: piecewise integration. With brackets: analytical estimates [Eq. (3) for model A and Eq. (4) for model B, respectively].

\begin{tabular}{lcc}
\hline \hline Model & Horizontal & Vertical \\
\hline $\mathrm{A}$ & $+0.0260(+0.0100)$ & $-0.0229(-0.0177)$ \\
$\mathrm{B}$ & $+0.0080(+0.0080)$ & $-0.0067(-0.0081)$ \\
\hline
\end{tabular}

the skin depth [10]. As expected, the resultant field decays exponentially rather than as $z^{-1 / 2}$ for $z>2 Z_{0} d^{2} /\left(\pi^{2} \rho_{R}\right)$. Keeping only the leading term in the Taylor series, the derived tune shift formula does not depend on the resistivity of the chamber wall as in the formula of model A [Eq. (3)], but has an explicit linear dependence on the chamber wall thickness $d$ :

$$
\frac{d \nu_{x, y}}{d I}= \pm D_{2 x y} \frac{C\left\langle\beta_{x, y}\right\rangle}{4 \pi} \frac{Z_{0}}{E / e} \cdot \frac{d}{\pi b^{3}},
$$

where $D_{2 x y}$ denotes the geometric factor for a given chamber cross section mentioned earlier and $\left\langle\beta_{x, y}\right\rangle$ represents an average beta value around the ring.

It must be noted that though both tune shift formulas in Eqs. (3) and (4) do not explicitly depend on the resistivity $\rho_{R}$, they are only valid for resistive walls and cannot be applied to chambers with perfectly conducting walls.

With the developed code, the overall incoherent focusing felt by a particle was calculated for each vacuum chamber with a database composed of a realistic chamber configuration around the ring with $\frac{\partial W_{x}}{\partial x_{w}}, \rho_{R}, d$ and local $\left\langle\beta_{x, y}\right\rangle$. The program treats an arbitrary beam filling of any bunch intensities. A particle relative time distance to any circulating bunch is taken into account rigorously over multiple turns since passage of a bunch to compute the sum of timedecaying fields in a given chamber according to each model. The incoherent tune shifts evaluated in two ways are compared at a beam current of $500 \mathrm{~mA}$ in the 4/4 filling (Table II): one using Eqs. (3) and (4), and the other, via piecewise integration around the ring. In the former, the quantities such as $b^{-2}$ and $b^{-3}$ were averaged around the ring. Reflecting the strong focusing of the SOLEIL optics, the analytical results indicate that the averaging used in the formulas is not precise enough. In particular, the smooth approximation employed in Eq. (3) to replace $\left\langle\beta_{x, y}\right\rangle$ by $R \nu_{x, y}^{-1}$ is found to enhance the discrepancies. Though the approximation is known to be valid for focus/defocus (FODO) cells, it breaks down for double-bend cell optics like SOLEIL. In fact, $\left\langle\beta_{x, y}\right\rangle$ are respectively 8.8 and $8.6 \mathrm{~m}$ horizontally and vertically, while $R \nu_{x}^{-1}=3.1 \mathrm{~m}$ and $R \nu_{y}^{-1}=5.5 \mathrm{~m}$. Hereafter, the more accurate integration scheme shall be compared with measurement.

In addition to the interbunch fields, it is important to take into account the self-field for a high intensity bunch due to 
the strong short-range resistive-wall fields. Let $k_{\mathrm{eff}}^{\text {bunch }}(\tau)$ be the effective focusing strength felt by a particle sitting at the relative time coordinate $\tau$ in the bunch. The average focusing strength $\left\langle k_{\text {eff }}^{\text {bunch }}\right\rangle$ felt by a particle in a bunch is then defined as the weighted average of $k_{\mathrm{eff}}^{\mathrm{bunch}}(\tau)$ by the bunch charge density $\rho(\tau)$ normalized to the total bunch charge $Q$. Having seen that parallel plates are a good approximation for our vacuum chambers (Table I), we shall use the relation $\frac{\partial}{\partial x_{w}}+\frac{\partial}{\partial x_{s}}=0\left(x_{s}\right.$ : source particle coordinate) valid for this case and rewrite $k_{\mathrm{eff}}^{\text {bunch }}(\tau)$ as

$$
\begin{aligned}
k_{\mathrm{eff}}^{\mathrm{bunch}}(\tau) & =\frac{1}{E / e} \int_{-\infty}^{\tau} \frac{\partial W_{x}}{\partial x_{w}}\left(\tau-\tau^{\prime}\right) \cdot \rho\left(\tau^{\prime}\right) d \tau^{\prime} \\
& =\frac{-1}{E / e} \int_{-\infty}^{\infty} \frac{\partial W_{x}}{\partial x_{s}}\left(\tau-\tau^{\prime}\right) \cdot \rho\left(\tau^{\prime}\right) d \tau^{\prime},
\end{aligned}
$$

where $\frac{\partial W_{x}}{\partial x_{s}}$ is the usual coherent transverse dipole wake function and using the causality to extend the integral to plus infinity. Expressing the two parts of the integrand in the second equation above as integrals of their Fourier transforms and using that expression for $k_{\mathrm{eff}}^{\mathrm{bunch}}(\tau)$ to get $\left\langle k_{\text {eff }}^{\text {bunch }}\right\rangle$, we have

$$
\left\langle k_{\mathrm{eff}}^{\text {bunch }}\right\rangle=\frac{4 \pi / Q}{E / e} \int_{0}^{\infty} \operatorname{Im} Z_{x}^{R W}(\omega) \cdot|\tilde{\rho}(\omega)|^{2} d \omega\left(\mathrm{m}^{-2}\right),
$$

where $Z_{x}^{R W}(\omega)$ is the horizontal dipolar resistive-wall impedance per unit length in $\Omega \mathrm{m}^{-2}$ and $\tilde{\rho}(\omega)$ is the Fourier transform of the bunch density $\rho(\tau)$. Evaluating $\left\langle k_{\mathrm{eff}}^{\text {bunch }}\right\rangle$ in the frequency domain has the advantage of utilizing the analytical impedance formulas for chambers having layers of metallic coating, such as that obtained by Burov and Lebedev [6]. The influence of a surface metallic layer such as NEG coating [11] will be discussed in Sec. V.

\section{ANALYSIS OF MEASURED TUNE SHIFTS AND ORBIT RESPONSE MATRIX IN 4/4 MULTIBUNCH FILLING}

In this section we explore the long-range aspect of the resistive-wall field in multibunch fillings. The variation of betatron tunes was measured for several beam currents between 0 and $500 \mathrm{~mA}$ in 4/4 filling (Fig. 2). As expected, the horizontal and vertical tunes increase and decrease linearly with current respectively. The tune shifts are found to be comparable in magnitude in both planes. The coherent tune shifts measured under the same condition for a single bunch at the corresponding bunch intensities are negligible compared to the observed variations. The concerned focusing occurs mainly in the medium and long SSs where the vertical aperture is smaller. The effective $\beta$ values over

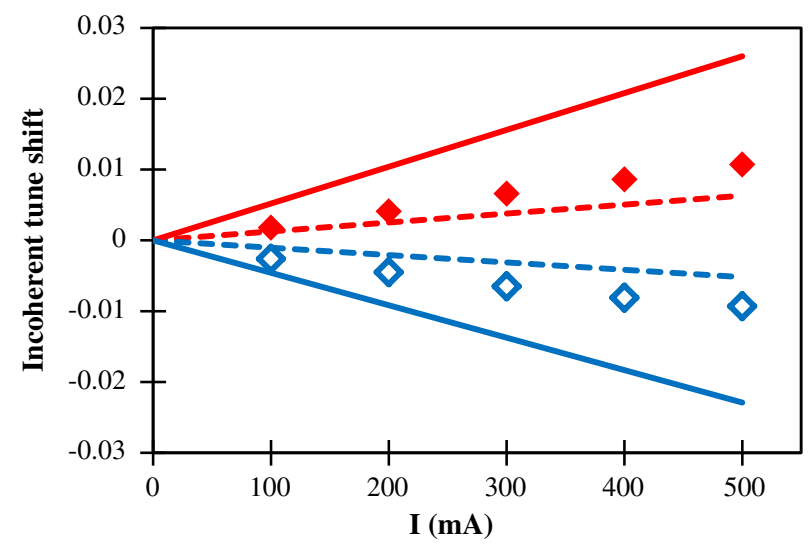

FIG. 2. Incoherent horizontal (red) and vertical (blue) tune shifts versus beam current in 4/4 filling: measured (diamond), model A (solid line), model B (dashed line). The precision on tune measured values is $1 \times 10^{-4}$. See the text for details of the calculations.

both types of SS are found to be nearly equal in the two planes: $5.8 \mathrm{~m}$ in the horizontal versus $5.1 \mathrm{~m}$ in the vertical.

A comparison between measured and calculated tune shifts is made as a function of total beam current (Fig. 2). The effect of field diffusion was taken into account explicitly as a function of the aperture, wall thickness, resistivity and time. The wall thickness $d$ was evaluated at $x=0$ where the image current is peaked. The predicted tune shifts are larger than the measured ones by roughly a factor of 2 with model A [2], while model B [10] underestimates them on the contrary by around $20 \%-30 \%$. Since the difference between the two models consists in the field penetration across finite thickness walls, the discrepancies should be attributed to the long-range field decay in vacuum chambers.

There are six $1.8 \mathrm{~m}$ long in-vacuum insertion devices (IDs) in the SOLEIL ring that are mechanically identical and are all located in the short SSs. Five of them are U20 undulators (20 mm period) and one is a WSV50 wiggler (50 mm period). Their minimum magnetic gap of $5.5 \mathrm{~mm}$ corresponds to a $5 \mathrm{~mm}$ physical gap due to magnetic shimming and metallic coating on the wall surface which protects the permanent magnets. When an in-vacuum ID is closed to its minimum gap with a high beam current, the resulting measured tune shifts are not only due to the incoherent wakefields, but also to the intrinsic focusing arising from the magnetic field. The intrinsic tune shifts were hence measured at low beam current and subtracted from the total ones recorded at high current in order to extract the measured incoherent contribution. The variation of this incoherent contribution with beam current is shown in Fig. 3 in the case of only one in-vacuum undulator is closed to its minimum gap. The quadrupolar type effect is seen to occur mainly in the horizontal plane in accordance with the larger value of the $\beta$ function at the undulator location in the corresponding plane. In the vertical plane, on the other hand, the quadrupolar effect is negligible, only 


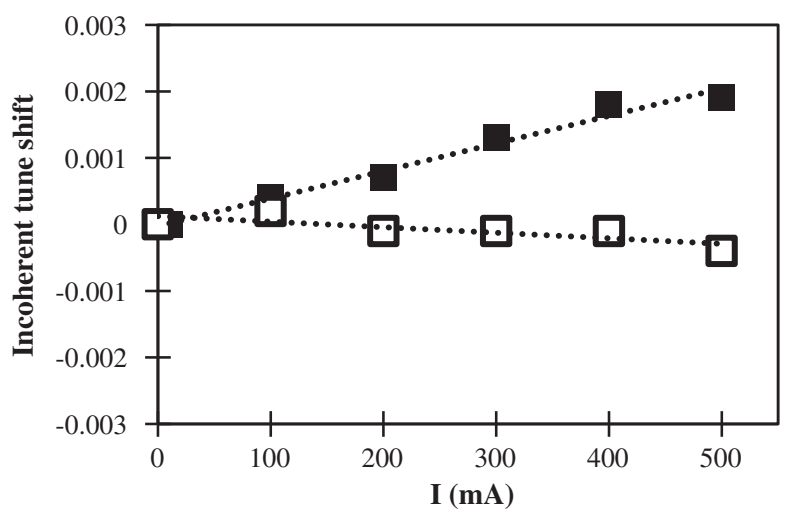

FIG. 3. Measured tune shifts due to a single in-vacuum ID (closed to minimum gap) versus beam current in 4/4 filling: black, horizontal; white, vertical; dotted lines, linear fittings.

slightly larger than the tune measurement precision of around $2 \times 10^{-4}$.

The impact of incoherent focusing on optics symmetry was measured at several beam currents for the bare machine as well as when the in-vacuum IDs are closed to their minimum gap. The standard orbit response matrix method measuring the effect of 57 dipolar correctors on the beam position at 122 beam position monitors (BPMs) in the transverse planes was used. As each matrix element depends on the $\beta$-functions at BPMs and correctors, the difference between measured and theoretical response matrices allows us to deduce the $\beta$ value deviation with respect to the model. Experimental data were analyzed using the LOCO code [14] according to the following steps: the machine model is varied to fit the measured response matrix by using quadrupoles and taking into account the measured dispersion function and the BPM noise. The variables used for the fit are the strengths of all the existing (163) quadrupoles in the ring and the BPM gains. The $\beta$-beating between the measured and the model optics is then calculated using the fitted model. By applying the calculated quadrupole strengths on the machine, the sources that generated the $\beta$-beating are compensated and the optics symmetry is restored in principle.

Regarding the incoherent wakefields, this type of analysis is suitable for identifying the location and the strength of the quadrupole-like sources. To this end, virtual quadrupoles were additionally introduced in the lattice model at the center of each of the 24 SSs to represent the incoherent focusing strength generated by the flat vacuum chambers. The LOCO analysis was then carried out using these new 24 quadrupoles.

The measurement of response matrix was made at two beam currents: 300 and $500 \mathrm{~mA}$ in the $4 / 4$ filling. All the in-vacuum undulators were open at maximum gap. The $\beta$-beating calculated from LOCO at $300 \mathrm{~mA}$ is $\pm 0.8 \%( \pm 1 \%)$ in the horizontal (vertical) plane. The corresponding distribution of the 24 quadrupole gradients around the ring (Fig. 4) clearly shows that the larger

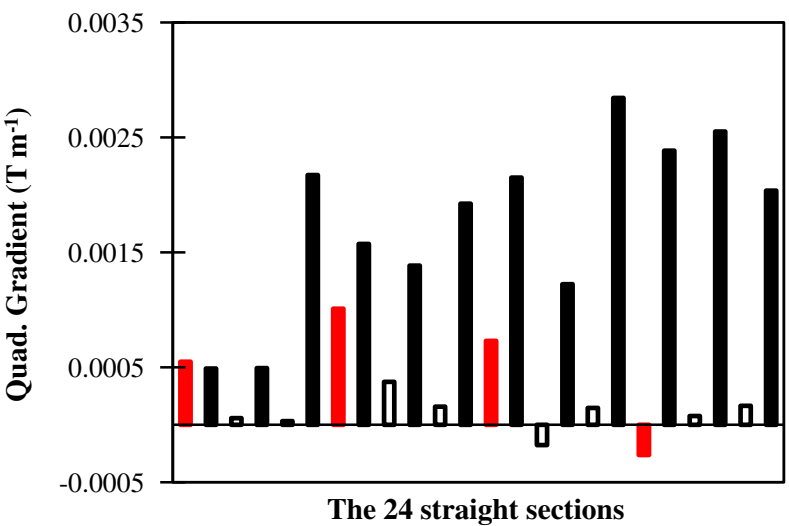

FIG. 4. Equivalent quadrupole gradient calculated by LOCO (300 $\mathrm{mA}$ case) for each of the 24 straight sections: long (red), medium (black) and short (white).

gradients are located at both medium and long SSs (cf. Table I). There are however exceptions: three medium SSs and one long SS that do not contribute to the incoherent focusing. They in fact correspond well to those straight sections either possessing large gap vacuum chambers or accommodating cryomodules for the rf cavities. The measurement and analysis were repeated at $500 \mathrm{~mA}$. A comparison of the gradients deduced from the measurements at 300 and $500 \mathrm{~mA}$ for each of the 24 quadrupoles showed ratios which were well constant and very close to that of the two currents as expected.

Another set of measurement was made at $300 \mathrm{~mA}$ with six in-vacuum IDs closed at their minimum gap of $5.5 \mathrm{~mm}$. As expected, LOCO now identifies quadrupolar components in the short SS where the undulators are located. Here, the deduced gradients are much larger than for the bare machine as the magnetic focusing is added to the incoherent one, as mentioned earlier. The total tune variation measured with in-vacuum IDs closed shows a strong vertical focusing due mainly to the magnetic effect. To extract the incoherent part, the measurement was repeated at low current (18 mA in the same 4/4 filling) to quantify the magnetic field contribution. The magnetic focusing occurs in both planes because of imperfections in the IDs, with corresponding tune shifts of +0.0079 and +0.0172 respectively in horizontal and vertical planes.

Subtracting the magnetic contributions, the tune shift due to incoherent wakes of the IDs is found to be +0.0058

TABLE III. Incoherent tune shifts deduced from the measurements at $300 \mathrm{~mA}$.

\begin{tabular}{lcc}
\hline \hline Contributor & Horizontal & Vertical \\
\hline $\begin{array}{l}\text { Low gap chambers in medium } \\
\text { and long SSs }\end{array}$ & +0.0066 & -0.0068 \\
$\begin{array}{l}\text { Six in-vacuum IDs closed to } \\
\text { their minimum gap }\end{array}$ & +0.0058 & -0.0010 \\
\hline \hline
\end{tabular}


TABLE IV. Comparison of effective gradients $\left(\mathrm{T} \mathrm{m}^{-1}\right)$ deduced from the measurements at a beam current of $300 \mathrm{~mA}$ with predicted values (model A) for each type of vacuum chamber.

\begin{tabular}{lcc}
\hline \hline Contributor & $\begin{array}{c}\text { Measured } \\
\text { gradient }\end{array}$ & $\begin{array}{c}\text { Calculated } \\
\text { gradient }\end{array}$ \\
\hline Long SS & +0.0009 & +0.00101 \\
Medium SS & +0.0018 & +0.00197 \\
In-vacuum IDs closed at & +0.0043 & +0.00789 \\
$\quad$ minimum gap & & \\
\hline \hline
\end{tabular}

$(-0.0010)$ in the horizontal (vertical) plane. Because the horizontal $\beta$ values at the IDs are much larger than the vertical $\beta$ values, the incoherent tune shifts occur mainly in the horizontal plane, and their magnitude is found to be comparable to that due to small gap chambers. This signifies that the horizontal contribution of the $5 \mathrm{~mm}$ physical gap in-vacuum ID over a total length of $10.8 \mathrm{~m}$ is comparable to the contribution of the small gap chambers over a total length of $71.6 \mathrm{~m}$. Table III summarizes the different tune shift contributions measured at a beam current of $300 \mathrm{~mA}$.

Experimentally, it is not possible to distinguish the effects of long and medium SSs for the bare machine. The data analysis using LOCO is therefore an effective alternative to independently estimating the effective incoherent focusing strength of each type of chamber or an invacuum undulator. Table IV compares the effective gradient deduced from the LOCO analysis and the one expected from calculations on the basis of Eqs. (2) and (3) using the formalism of model A [2]. On the order of magnitude, the agreement is fairly good for the three types of chamber. In particular, the ratio between the effective gradients of long and medium SS chambers is exactly equal to the ratio of the square of the inner vertical aperture values (i.e. a factor of 2). As expected from the calculations, the contribution of one in-vacuum undulator at minimum gap is much larger due naturally to the smaller inner vertical aperture.

\section{IMPACT OF INCOHERENT FOCUSING ON NEIGHBORING BUNCHES}

Due to collective effects, a high current bunch likely exhibits coherent tunes that are significantly different from the ones defined by the machine optics. To measure the latter a small current (parasitic) bunch, of the order of $10 \mu \mathrm{A}$, is therefore stored sufficiently far away from the high current bunch so as not to be influenced by the wakefield of the latter, and is selectively excited by a bunch-by-bunch transverse feedback (TFB) system [15]. Indeed, to investigate the time dependence of the wakes excited by the main bunch, the tunes of the parasitic bunches were measured as a function of their distance from the high current bunch. The tune shifts described hereafter are defined as the difference between the incoherent tunes measured on parasitic bunches and the zero-current machine tunes measured at a very low beam current prior to the experiment.

It is anticipated that the incoherent wake excited by the main bunch damps rapidly after its passage leaving the long-range tail that scales like $t^{-1 / 2}$ where $t(=z / c)$ denotes the time since the bunch passage [cf. Eq. (2)]. Only four parasitic bunches were therefore stored close to the main bunch, upstream and downstream. Though our major concern is to follow the possible tune variations along the four downstream bunches, the measurement of the upstream bunches was added to verify our entire setup. TFB was employed to excite each of the eight parasitic bunches independently to measure their tunes. Since the tune detection of TFB is limited to a bunch of higher than $10 \mu \mathrm{A}$, the current of the eight bunches was fixed to $15 \mu \mathrm{A}$ each, which is nearly $10^{3}$ times smaller than the main bunch current.

Storing a high current bunch also requires the action of TFB to stabilize it against head-tail instability without increasing the chromaticity. Under the nominal setup with a BPM as a detector, when the intensity of the main bunch was high, its signal extended non-negligibly in time over to the adjacent downstream bunch, whose tunes then became difficult to detect. A significant improvement resulted in the detection by replacing the BPM by a shorted stripline which minimized the cross talk. In the new setup, the measurement precision was within $\pm 5 \times 10^{-4}$ horizontally and $\pm 6 \times 10^{-4}$ vertically. The mean values over ten readings were recorded.

Incoherent tune shifts were measured for the eight parasitic bunches mentioned above at three values of the main bunch current: 12,16 and $20 \mathrm{~mA}$. The results showed fairly good proportionality with the bunch intensity. For all the parasitic bunches, the measured tune shifts were positive horizontally and negative vertically. As expected, the tune shifts measured for the four upstream bunches were more or less equal for all three currents of the main bunch, while much larger variations were found for the four downstream ones with the first $(+1)$ adjacent to the main bunch showing the maximum values. It was in fact our main concern to see if the bunch +1 experiences the largest tune shifts among the eight since it should suffer from the largest amplitude of the wake induced by the main bunch. The mean tune shifts of the eight bunches, which should depend on the long-range field, turned out to have the expected relative magnitudes and signs that match the current of the main bunch, even though they were less than $5 \times 10^{-4}$ and small.

The measured incoherent tune shifts were compared with expectations for a $20 \mathrm{~mA}$ main bunch (Fig. 5). With model A, we observe first that the discrepancy is amplified by the too large values of the overall offsets, related to the longrange field, in both planes. Their values are measured for bunches located upstream the main bunch. Namely, model A appears to overestimate the long-range field again as in 


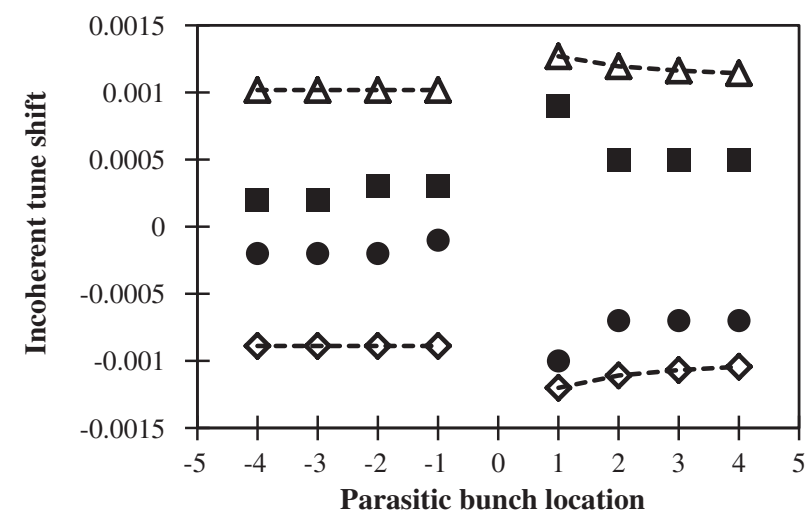

(a)

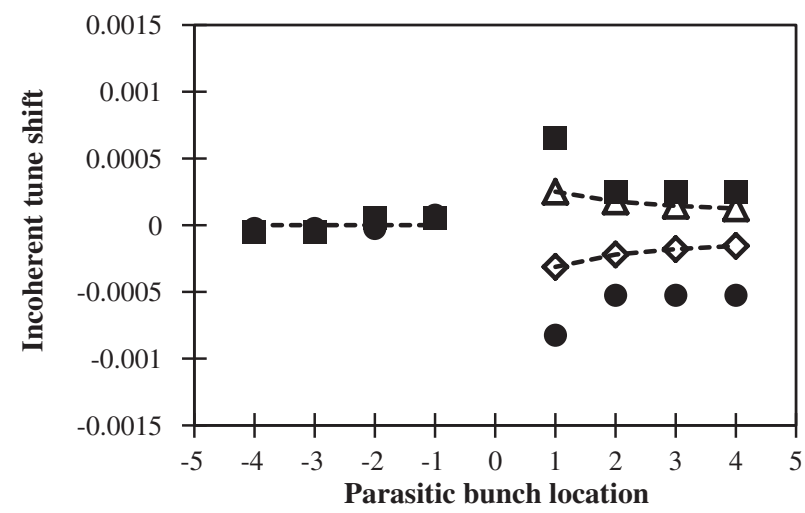

(b)

FIG. 5. Horizontal (black square) and vertical (black circle) tune shifts measured on the eight parasitic bunches for a $20 \mathrm{~mA}$ main bunch. Horizontal (white triangle) and vertical (white diamond) incoherent tune shifts calculated with model A. (a) Raw data. (b) With mean long-range tune shifts of the upstream bunches subtracted.

the multibunch case (cf. Fig. 2). With model B, on the other hand, these offsets are 0.00032 and -0.00027 horizontally and vertically respectively, which are more or less in agreement with the observed ones [Fig. 5(a)]. If we compare the measured relative tune shifts of the parasitic bunches to the expectation by removing the offsets, the relation is inverted and the calculations considerably underestimate them [Fig. 5(b)]. To be noted is that these relative tune shifts of parasitic bunches should not depend on the long-range diffusion process. Apart from the above quantitative discrepancies, the calculation well reproduces qualitatively the relative tune shifts as a function of bunch locations.

\section{SHORT-RANGE INTRABUNCH CHARACTERISTICS}

The third important range to explore is the incoherent field experienced by an electron in an intense bunch. This feature raises a particular concern to machines such as SOLEIL delivering high bunch intensity modes, since electrons may effectively see optics that are considerably distorted, which must then be taken into account in the machine tuning. Below we shall pursue it by attempting to measure the incoherent tunes of an intense bunch.

With such a bunch current, however, it is generally difficult to identify the measured tunes as its incoherent ones, due likely to strong coherent effects arising from dipolar wakes. Hence we shall set up an experiment that would allow measuring the incoherent tunes without being affected by the coherent ones. The idea is to make use of a single particle resonance driven by the incoherent fields arising from magnets and monopolar wakes. Specifically, we shall work with the betatron coupling resonance $\nu_{x}-\nu_{y}=8$, which is sufficiently close to the machine tunes $(18.155,10.229)$. There is an advantage of detecting accurately the resonance condition by following the maximum vertical beam size with an $\mathrm{x}$-ray pinhole camera, without incurring beam losses. The disadvantage is that with the use of a coupling resonance condition alone, we are not able to deduce the tune shift in each transverse plane separately. Nevertheless, our studies up to now have justified both theoretically and experimentally that the two tune shifts can be assumed approximately equal in magnitude with opposite signs under the specific SOLEIL optics employed.

The measurement was carried out on the bare machine with all the in-vacuum undulators open at maximum gap. The first step was to minimize the width of the coupling resonance with skew quadrupoles so to bring the two tunes as close as possible. The measurement was first performed with a small current per bunch (18 mA in 312 bunches). The distance between the two fractional parts was varied with a pair of quadrupole families by keeping the vertical tune fixed to its initial value while the horizontal one is increased to approach the vertical (tunes are measured with a precision of $1 \times 10^{-4}$ ). The tunes cross each other when their difference gets smaller than $1.7 \times 10^{-3}$. The variation of vertical emittance was simultaneously followed (Fig. 6, in black). Zooming into the region close to resonance, the maximum vertical emittance is obtained only when the two tunes are on resonance with a precision of better than $5 \times 10^{-4}$. The vertical beam size measured with our $\mathrm{x}$-ray pinhole camera is therefore confirmed to be a good diagnostic in identifying a beam on the coupling resonance.

The same step was repeated for a $20 \mathrm{~mA}$ bunch. A very small current $(10 \mu \mathrm{A})$ bunch was stored simultaneously at the opposite rf bucket in the ring to keep track of the machine tunes measured by TFB. The horizontal tune was varied exactly in the same way as in the previous step to reach the coupling resonance and the vertical emittance of the high current bunch was recorded simultaneously (Fig. 6, in red). In the resonance region we clearly observe in this case that the vertical emittance is maximized when the machine tunes are still far from the coupling resonance. The above indicates that the incoherent tunes of the high current bunch are shifted with respect to the previous case. 


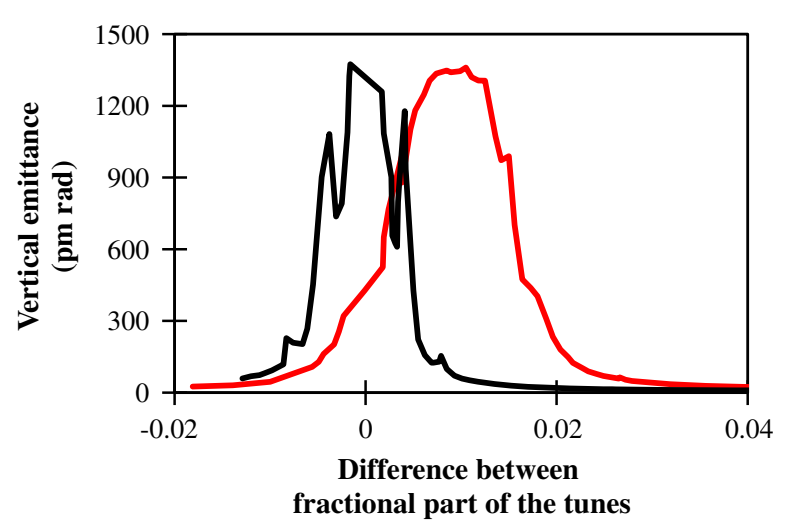

FIG. 6. Variation of the measured vertical emittance as a function of the distance between the transverse tunes. Black: low current per bunch. Red: $20 \mathrm{~mA}$ current per bunch.

The high current bunch reaches the resonance when the machine tunes are separated by $10 \times 10^{-3}$. Since we may assume that $\Delta \nu_{x} \approx-\Delta \nu_{y}$, it follows that value of $10 \times 10^{-3}$ represents $2 \cdot \Delta \nu_{x}$, namely $\Delta \nu_{x}=-\Delta \nu_{y}=5 \times 10^{-3}$.

The experiment was extended to four other bunch currents (3, 6, 12 and $16 \mathrm{~mA}$ ) and compared (Fig. 7). As expected, the tune shifts are seen to diminish with lower current. On the other hand, the range of tunes where the vertical emittance is at maximum is enlarged with higher current. This is supposedly due to the electrons in an intense bunch having larger tune spreads, above all due to an increased energy spread since the threshold for the longitudinal turbulent regime is known to be at around $8 \mathrm{~mA}$ from other measurements [16]. The resonance condition is hence met for a larger range of tunes. For the $3 \mathrm{~mA}$ case, since the tune shift becomes comparable to the resonance width, the maximum vertical emittance is defined less precisely. In all other cases, the position of the maximum vertical emittance could be defined with a precision of better than $5 \times 10^{-4}$.

Discussing now the expectations, since the multiturn fields are found to represent merely a few percent of the

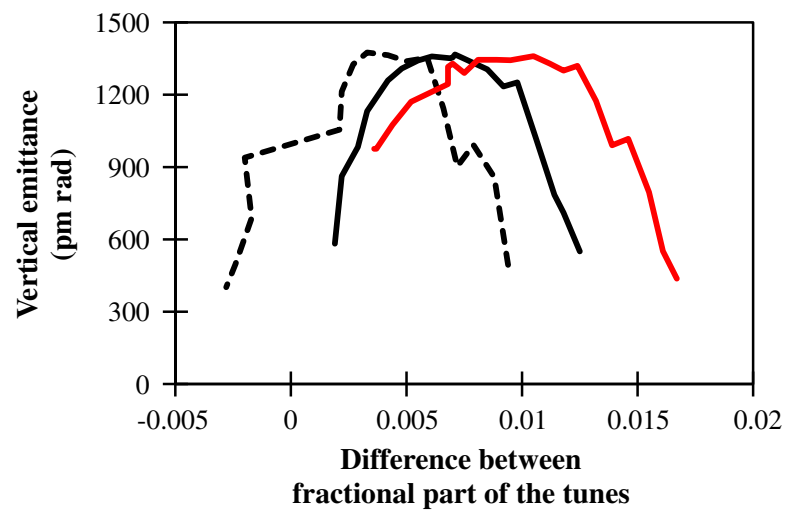

FIG. 7. Same as Fig. 6 for bunch currents: 20 (red), 12 (black) and $6 \mathrm{~mA}$ (black dashed). direct short-range contribution, they were discarded. The tune shifts were therefore evaluated exclusively using $\left\langle k_{\text {eff }}^{\text {bunch }}\right\rangle$ felt by an electron in a bunch in a flat chamber, as given by Eq. (6). Second, as the latter involves the imaginary part of the horizontal dipolar resistive-wall impedance $\operatorname{Im} Z_{x}^{R W}(\omega)$, which is known to be affected by a metallic layer on the wall surface $[6,11,17]$, the NEG coating was taken into account in the computation. It must be noted that nearly half of the vacuum chambers in the SOLEIL ring are NEG coated. In particular, it was previously found that the coating thickness of $1 \mu \mathrm{m}$ and assuming the resistivity of $500 \times 10^{-8} \Omega \mathrm{m}$ for NEG increase $\operatorname{Im} Z_{x}^{R W}(\omega)$ by nearly a factor of 2 over the frequency range seen by an electron bunch [11].

Though the coating thickness was chosen to be $1 \mu \mathrm{m}$, in reality the thickness on the surface just above and below the beam path, namely the parts of the chamber walls closest to the beam, was reduced to $0.5 \mu \mathrm{m}$ at SOLEIL in the aim of avoiding an anomalous increase of the impedance such as observed at Elettra with NEG coated chambers [17]. It was later estimated, however, that multilayer impedance models do not explain the magnitude of the increase observed at Elettra [11]. Other impedance sources such as surface roughness arising from the coating may be suspected as the origin. Confirming the previous findings [11] that dependence of $\left\langle k_{\text {eff }}^{\text {bunch }}\right\rangle$ on the resistivity of NEG as well as on the thickness of the NEG layer is small, the resistivity of $200 \times 10^{-8} \Omega \mathrm{m}$ was assumed for NEG and the thickness was varied between 0.5 and $1 \mu \mathrm{m}$ in the following computations.

The horizontal incoherent tune shifts computed as above are compared with the measured data (Fig. 8). While the band covered between the two limiting coating thicknesses matches the measurement at low current rather well, the simple linear dependence on beam current fails in

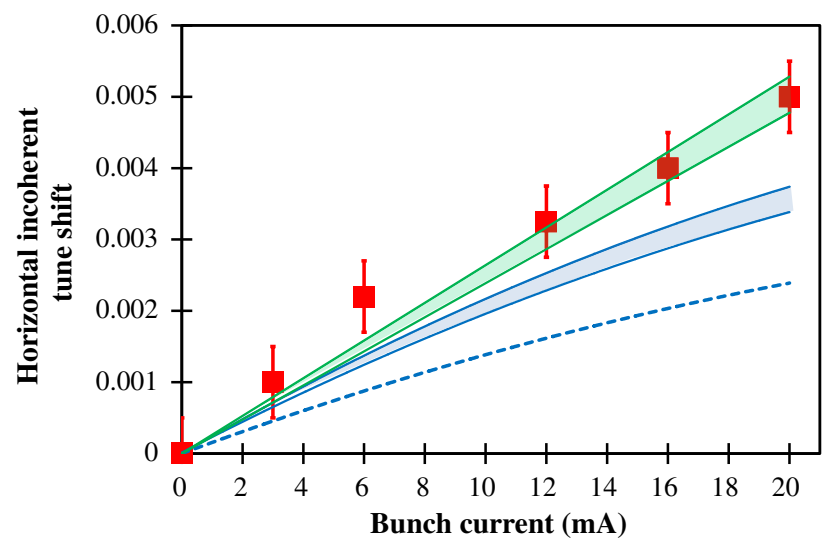

FIG. 8. Measured (red square) horizontal incoherent tune shift as a function of bunch current. Values expected from theory: without NEG coating and with bunch lengthening (blue dashed line); with NEG coating ( 0.5 and $1.0 \mu \mathrm{m}$ thickness) and with bunch lengthening (blue area); with NEG coating (0.5 and $1.0 \mu \mathrm{m}$ thickness) and without bunch lengthening (green area). 
reproducing the saturating trend seen in the measured data. The last is expected to arise from the bunch lengthening that effectively weakens the focusing, which is not taken into account in the computation. Here the effect of bunch lengthening was incorporated as follows: Assuming a Gaussian bunch, the effective resistive-wall impedance seen by the beam $\left|Z_{x}^{R W}\right|_{\text {eff }}$ is proportional to the square root of the rms bunch length $\sigma_{L}$ [18], to which $\left\langle k_{\mathrm{eff}}^{\text {bunch }}\right\rangle$ in Eq. (6) is related as $\left|Z_{x}^{R W}\right|_{\text {eff }} \cdot \sigma_{L}^{-1}$. Namely, $\left\langle k_{\text {eff }}^{\text {bunch }}\right\rangle$ scales as $\sigma_{L}^{-1 / 2}$. The original values of $\left\langle k_{\mathrm{eff}}^{\mathrm{bunch}}\right\rangle$ were rescaled by this relation using the measured bunch lengthening curve. The calculated tune shifts are now found to follow the measured values much better at high current, though they tend to underestimate the measured data. In particular, removing the effect of NEG coating, the agreement between the two is significantly deteriorated (Fig. 8).

\section{CONCLUSION}

In the present paper, we studied the incoherent wakefields arising from the passage of a high current beam in noncircular resistive-wall beam pipes experimentally and numerically for the SOLEIL storage ring. Experimentally, the effects were pursued in terms of incoherent betatron tune shifts and local focusing strengths extracted from the orbit response analysis. Numerically, the field excited in every vacuum chamber was calculated rigorously according to the corresponding cross section geometry using the formalism of Yokoya [1]. The effect of transverse focusing was then integrated around the ring by taking also into account the local variations of the beta functions. The time dependence of a decaying field was described according to the two models that both considered the field penetration across a finite thickness wall, developed respectively by Chao et al. [2] (model A) and Shobuda and Yokoya [10] (model B). A special effort was made to explore the time dependence of the excited field in the following three distinct regimes: (1) extremely long range over many revolutions; (2) intermediate range involving adjacent bunches; and (3) short range felt by particles within a bunch. Three specific experimental methods were applied respectively.

In the first long-range regime dealing with multibunch beams, the agreement with the measured tune shifts of the tune shift formula of model A [Eq. (3)] would be accidental as the smooth approximation employed is not valid for SOLEIL and the more rigorous calculation integrating the local focusing strength results in overestimation by nearly a factor of 2. The same computation with model B gave on the contrary an underestimation but with better agreement. The obtained results suggest that the reality lies somewhere between the two models. A unique attempt of extracting the effective focusing strength from orbit response analysis using LOCO managed to reproduce the overall low-gap chamber configuration of the ring along with the correct dependence on the total beam current. Though similar orbit analyses were made elsewhere in the past to measure the transverse kick factors [19-21], we believe that the present work is the first to probe locally the incoherent monopolar wakes instead of dipolar ones.

The attempt made in the second regime was to measure the betatron tunes of parasitic bunches of around $10 \mu \mathrm{A}$ each placed upstream and downstream a high current bunch, as a function of their distance from the latter and its intensity. A special application of bunch-by-bunch transverse feedback developed at SOLEIL was made for this measurement: It stabilizes the high intensity bunch against coherent instability while simultaneously exciting a selected parasitic bunch to measure its tunes. As expected, a clear asymmetry was observed in the tune shifts between upstream and downstream bunches, where the first downstream bunch closest to the high intensity bunch showed the largest tune shifts with the expected signs. Again model A overestimated the measurement, which originated in the common long-range offset involving field diffusion. On the contrary, calculations underestimated the relative tune shifts of the parasitic bunches, namely the shorter range contributions.

In the third intrabunch regime, a coupling resonance was utilized to measure the average incoherent tunes of the electrons in a high intensity bunch. As expected, much larger tune shifts than those in multibunch mode were measured. For the same total beam current, the horizontal tune shift is 18 times larger in a single bunch than in multibunch (Figs. 2 and 8). This could explain the different working points required for high current per bunch modes of operations as well as their much higher sensitivity to tunes. Inclusion of bunch lengthening in the computation much better described the saturating feature of the measured tune shift with current. It is worthwhile noting that in this regime where the long-range field diffusion plays no role, the calculation underestimates the measured ones as in the relative tune shifts of the parasitic bunches above. Besides, inclusion of the effect of NEG coating was found to significantly improve the agreement.

\section{ACKNOWLEDGMENTS}

The authors thank Laurent S. Nadolski for his helpful support in the data analysis using the LOCO code, Francis Cullinan for his contributions to the beam measurements as well as proof reading the manuscript and Amor Nadji, head of the Machine division, for his support. They also thank the machine operators for their general help during the experiments. The authors have also benefited from fruitful discussions during the EuCARD2 TWIICE workshop [22].

[1] K. Yokoya, Resistive wall impedance of beam pipes of general cross section, Part. Accel. 41, 221 (1993). 
[2] A. Chao, S. Heifets, and B. Zotter, Tune shifts of bunch trains due to resistive vacuum chambers without circular symmetry, Phys. Rev. ST Accel. Beams 5, 111001 (2002).

[3] A. Loulergue et al., Double low beta straight section for dual canted undulators at SOLEIL, in Proceedings of the International Particle Accelerator Conference, Kyoto, Japan (ICR, Kyoto, 2010), pp. 2496-2498.

[4] P. Brunelle et al., New optics for the SOLEIL storage ring, in Proceedings of the 2nd International Particle Accelerator Conference, San Sebastián, Spain (EPS-AG, Spain, 2011), pp. 2124-2126.

[5] R. L. Gluckstern, J. van Zeijts, and B. Zotter, Coupling impedance of beam pipes of general cross section, Phys. Rev. E 47, 656 (1993).

[6] A. Burov and V. Lebedev, Transverse resistive wall impedance for multilayer flat chambers, in Proceedings of the 8th European Particle Accelerator Conference, Paris, 2002 (EPS-IGA and CERN, Geneva, 2002), pp. 1455-1457.

[7] S. Heifets, Report No. SLAC-PUB-7985, 1998.

[8] R. Nagaoka, Impact of resistive-wall wakefields generated by low-gap chambers on the beam at the ESRF, in Proceedings of the 19th Particle Accelerator Conference, Chicago, IL, 2001 (IEEE, Piscataway, NJ, 2001), pp. 3531-3533.

[9] R. Nagaoka, J.-L. Revol, and P. Kuske, Incoherent transverse tune shifts due to resistive low-gap chambers, in Proceedings of the 8th European Particle Accelerator Conference, Paris, 2002 (Ref. [6]), pp. 1541-1543.

[10] Y. Shobuda and K. Yokoya, Resistive wall impedance and tune shift for a chamber with a finite thickness, Phys. Rev. E 66, 056501 (2002).

[11] R. Nagaoka, Study of resistive-wall effects on SOLEIL, in Proceedings of the 9th European Particle Accelerator Conference, Lucerne, 2004 (EPS-AG, Lucerne, 2004), pp. 2038-2040.

[12] N. Mounet and E. Métral, Generalized form factors for the beam coupling impedances in a flat chamber, in Proceedings of the International Particle Accelerator
Conference, Kyoto, Japan (ICR, Kyoto, 2010), pp. 2042-2044.

[13] H. Henke and O. Napoly, Report No. CERN-LEP-RF-8971, 1989.

[14] J. Safranek, G. Portmann, A. Terebilo, and C. Steier, MATLAB-based LOCO, in Proceedings of the 8th European Particle Accelerator Conference, Paris, 2002 (Ref. [6]), pp. 1184-1186.

[15] R. Nagaoka et al., Operational status of the transverse bunch by bunch feedback system at SOLEIL, in Proceedings of the International Particle Accelerator Conference, Kyoto, Japan (ICR, Kyoto, 2010), pp. 2746-2748.

[16] M.-A. Tordeux and M. Labat, Observation of bursting threshold with a bolometer (private communication); F. Cullinan and R. Nagaoka, Observation of a longitudinal instability through the transverse beam size, SOLEIL internal note, 2015.

[17] E. Karantzoulis, V. Smaluk, and L. Tosi, Broad band impedance measurements on the electron storage ring ELETTRA, Phys. Rev. ST Accel. Beams 6, 030703 (2003).

[18] R. Nagaoka and K. F. Bane, Collective effects in a diffraction-limited storage ring, J. Synchrotron Radiat. 21, 937 (2014).

[19] L. Emery, G. Decker, and J. Galayda, Local bump method for measurement of transverse impedance of narrow-gap ID chambers in storage rings, in Proceedings of the 19th Particle Accelerator Conference, Chicago, IL, 2001 (Ref. [8]), p. 1823.

[20] V. Sajaev, Transverse impedance distribution measurement using the response matrix fit method at APS, in Proceedings of the 2003 Particle Accelerator Conference, Portland, OR (IEEE, New York, 2003), pp. 417-419.

[21] T. Perron, L. Farvacque, and E. Plouviez, Vertical effective impedance mapping of the ESRF storage ring, in Proceedings of the 9th European Particle Accelerator Conference, Lucerne, 2004 (Ref. [11]), pp. 2053-2055.

[22] EuCARD2 TWIICE topical workshop: http://indico.cern .ch/event/277919/. 